BULLETIN OF THE

AMERICAN MATHEMATICAL SOCIETY

Volume 77, Number 3, May 1971

\title{
NOT EVERY 0-DIMENSIONAL REALCOMPACT SPACE IS $N$-COMPACT
}

\author{
BY PETER NYIKOS
}

Communicated by Steve Armentrout, September 8, 1970

Introduction. $N$-compact spaces were introduced by S. Mrówka in [1], where the general concept of an $E$-compact space was defined: given a Hausdorff space $E$, a space $X$ is E-compact if it is homeomorphic to a closed subspace of $E^{m}$ for some cardinal number $m$. Thus the $I$-compact spaces (where $I$ is the closed unit interval) are the compact Hausdorff spaces, the $R$-compact spaces are the realcompact spaces or $Q$-spaces, and the 2-compact spaces (where 2 represents the discrete space consisting of two points) are the 0-dimensional compact Hausdorff spaces. The $N$-compact spaces are those which can be embedded as closed subspaces in $N^{m}$ where $N$ is the set of natural numbers with the discrete topology.

In terms of category theory, the category of $E$-compact spaces forms the epireflective hull of $E$ in $T_{2}$, the category of Hausdorff spaces, i.e., the intersection of all replete reflective subcategories of $T_{2}$ containing the space $E$ (cf. [4]).

The main properties of $E$-compact spaces were given in [2], where it was asserted that the $N$-compact spaces are precisely the 0 -dimensional realcompact spaces ("0-dimensional" there, as here, means "having a base of clopen sets"). It is clear that every $N$-compact space is 0-dimensional and realcompact, but the proof of the converse in [2] was incomplete and indeed the validity of the converse remained an open question.

The first significant result in that direction was obtained by $\mathrm{H}$. Herrlich in [3], where he proved that every strongly 0-dimensional realcompact space is $N$-compact ( $X$ is strongly 0 -dimensional iff it is completely regular and $\beta X$ is totally disconnected). Attention then focused on the only known example of a 0 -dimensional realcompact space which is not strongly 0 -dimensional, a metrizable space $\Delta$ introduced by P. Roy in [5] and described in detail in [6]. The main result of this paper is that $\Delta$ is not $N$-compact; and so it is established that the class of $N$-compact spaces is properly contained in the class of

AMS 1970 subject classifications. Primary 54D30, 54G20; Secondary 54B10, 54C25, 54D60, 54E15, 54F50.

Key words and phrases. Clopen filter, countable intersection property, E-compact, $N$-compact, partition, realcompact, strongly 0-dimensional, 0-dimensional. 
0-dimensional realcompact spaces. Now the chief unsolved problem in this area is whether the $N$-compact spaces are all strongly 0 -dimensional.

All spaces are assumed to be Hausdorff.

1. $N$-compactness. Theorem 1 gives conditions equivalent to $N$-compactness. The equivalence of (i) and (iii) was first proved by Herrlich [3]. The full theorem was discovered independently by a number of workers, including K. Chew, I. Fleischer and G. Rejas, A. Hager, the author, and perhaps others.

Definition. Let $X$ be 0 -dimensional. For each ordinal $\alpha$ let $N_{\alpha}$ be the uniformity on $X$ whose base consists of all equivalence relations partitioning $X$ into $<\aleph_{\alpha}$ clopen sets.

Thus $N_{1}$ is the uniformity whose base consists of all equivalence relations partitioning $X$ into countably many clopen sets. Each equivalence relation may be looked at either as an entourage on $X \times X$, or as a two-valued pseudometric, with $d_{r}(x, y)=0$ iff $x$ $\equiv y \bmod r$, or as an open cover whose members are the clopen sets of the partition.

In what follows, a filter on the Boolean algebra of clopen subsets of a space $X$ will be called simply a clopen filter. A filter $F$ has the countable intersection property if $\bigcap_{i \in I} A_{i} \neq \varnothing$ for any countable index $I$; it is not required that $F$ be closed under countable intersection.

Theorem 1. Let $X$ be 0-dimensional. The following conditions are equivalent:

(i) $X$ is $N$-compact.

(ii) $X$ is complete under $N_{1}$.

(ii') $X$ is complete under $N_{\alpha}$ for any nonmeasurable cardinal $\boldsymbol{N}_{\alpha}>\boldsymbol{N}_{0}$

(iii) Every clopen ultrafilter on $X$ with the countable intersection property is fixed.

A proof that (i), (ii), and (iii) are equivalent will be found in [8].

2. Strong 0-dimensionality. Various conditions equivalent to strong 0 -dimensionality have been proven over the years, with the first three conditions of Theorem 2, below, already appearing in [7, pp. 245247], where the term "0-dimensional" is used for them. I follow [7, Chapter 15] in using $\mathcal{C}\left[\right.$ resp. $\left.\mathcal{C}^{*}\right]$ to denote the uniformity generated on the completely regular space $X$ by the real-valued [resp. the bounded real-valued] continuous functions.

This paper adopts the following definition of strong 0 -dimensionality: a strongly 0 -dimensional space is a completely regular space such 
that, given two disjoint zero-sets $Z_{1}$ and $Z_{2}$, there exists a clopen set $C$ such that $Z_{1} \subset C$ and $Z_{2} \cap C=\varnothing$. This is probably the most satisfactory internal characterization of these spaces and leads immediately to the result that every strongly 0 -dimensional space $X$ is 0 -dimensional. (Given $x \in X$ and an open set $U$ containing $x$, choose a continuous $f: X \rightarrow R$ such that $f\left(U^{c}\right)=0, f(x)=1$. Let $Z_{1}$ be $f^{-1}(\{1\})$ and $Z_{2}=f^{-1}(\{0\})$; we thus obtain a clopen set $C \subset U$ such that $x \in C$.) The following theorem establishes the validity of the characterization given in the introduction.

THEOREM 2. Let $X$ be 0-dimensional. The following conditions are equivalent:

(i) $X$ is strongly 0 -dimensional.

(ii) $\beta X$ is totally disconnected.

(iii) Every finite cover of $X$ by cozero-sets can be refined to a partition of $X$ into finitely many clopen sets.

(iii') Every countable cover of $X$ by cozero-sets can be refined to $a$ partition of $X$ into countably many clopen sets.

(iv) Every zero-set is a countable intersection of clopen sets.

(v) $\mathfrak{e}=N_{1}$.

$\left(\mathrm{v}^{\prime}\right) \mathfrak{e}^{*}=N_{0}$.

Furthermore, conditions (i) through (iv) are equivalent for any completely regular space.

This full theorem has been known to I. Fleischer and G. Rejas, A. Hager, and possibly others.

Using the equivalence of (i) and ( $v$ ) and the fact that completeness under $\mathcal{C}$ is equivalent to realcompactness (cf. [7, p. 226]), we obtain the following

CoROLLARY. Every strongly 0-dimensional realcompact space is $N$ compact.

This Corollary was first proven by H. Herrlich in [3] using different methods. (However, interestingly enough, this Corollary was already contained implicitly in [2, p. 431].)

3. P. Roy's space is not $N$-compact. The main result of this paper is Theorem 3, which establishes proper containment of the category of $N$-compact spaces in the category of 0 -dimensional realcompact spaces. The space used is $\mathrm{P}$. Roy's space $\Delta[5],[6]$, which is metrizable $[6, p .125]$ and has the cardinality of the continuum, and so is realcompact (cf. [7, p. 232]). It is also 0-dimensional [6, p. 127] and, in view of $[6$, Lemma 5.9] and the fact that every closed set in a metric space is a zero-set, it is not strongly 0-dimensional. Furthermore, 
THEOREM 3. $\Delta$ is not $N$-compact.

To prove this, we exhibit $2^{N_{0}}$ distinct free clopen ultrafilters on $\Delta$, each of them having the countable intersection property. Unfortunately, $\Delta$ is a very complicated space, and the definition of any of these ultrafilters requires one to lean heavily on the whole description of $\Delta$ found in [6]. Due to lack of space the definition will not be given here, but a future paper will contain both a definition of these ultrafilters and a proof of their essential properties.

Very roughly speaking, the simplest of these free clopen ultrafilters (of course, any one of them is sufficient for establishing Theorem 3) has as members all clopen sets that take up a big chunk of $\Delta$. These "fat" clopen sets form a clopen ultrafilter with the countable intersection property (indeed, the $m$-intersection property for any cardinal $m<2^{\aleph_{0}}$ ) because of a radically uneven split: given any clopen set $A$, either it or its complement (but not both) is "fat," and each fat one takes up so much of $\Delta$ that even $m$ of the "thin" sets $\left(m<2^{\aleph_{0}}\right)$ will not cover all of $\Delta$. Freeness follows from the fact that, loosely speaking, a clopen set cannot take up a big chunk of $\Delta$ and yet have a diameter much smaller than that of $\Delta$ itself, regardless of the metric that is chosen for $\Delta$. And since $\Delta$ is 0 -dimensional it follows that a fixed clopen ultrafilter must contain sets of arbitrarily small diameter.

4. The other containment. The major remaining problem was mentioned in the Introduction and may be simply restated as follows: is every closed subspace of $N^{m}$ strongly 0-dimensional for all cardinals $m$ ? If the answer is affirmative, it would immediately follow that the category of strongly 0 -dimensional realcompact spaces is closed under arbitrary products and under the operation of taking a closed subspace, for the category of $N$-compact spaces is thus closed. And it is not even known whether the product of two strongly 0-dimensional spaces is strongly 0-dimensional; indeed, as far as the author knows, it is not known whether the Sorgenfrey plane is strongly 0-dimensional, even though the Sorgenfrey line is strongly 0-dimensional, being Lindelöf (cf. [7, p. 247]).

\section{BIBLIOGRAPHY}

1. S. Mr6wka, On universal spaces, Bull. Acad. Polon. Sci. Cl. III 4 (1956), 479481. MR 19, 669.

2. R. Engelking and S. Mrówka, On E-compact spaces, Bull. Acad. Polon. Sci. Sér. Sci. Math. Astronom. Phys. 6 (1958), 429-436. MR 20 \#3522.

3. H. Herrlich, (E-kompakte Ruume. II, Mathematisches Institut der Freien Universität Berlin, 1965; reprinted, Math. Z. 96 (1967), 228-255. MR 34 \#5051.

1968.

4. - - Topologische Reflexionen und Coreflexionen, Springer-Verlag, Berlin, 
5. P. Roy, Failure of equivalence of dimension concepts for metric spaces, Bull. Amer. Math. Soc. 68 (1962), 609-613. MR 25 \#5495.

6. - Nonequality of dimensions for metric spaces, Trans. Amer. Math. Soc. 134 (1968), 117-132. MR $37 \# 3544$.

7. L. Gillman and N. Jerison, Rings of continuous functions, University Series in Higher Math., Van Nostrand, Princeton, N. J., 1960. MR 22 \#6994.

8. Kim-Peu Chew, A characterization of $N$-compact spaces, Proc. Amer. Math. Soc. 26 (1970), 679-682.

Carnegie-Mellon University, Pittsburgh, Pennsulvania 15213 